\title{
BMJ Open Association between vaping and health outcomes in patients with opioid use disorder: a systematic review protocol
}

\author{
Nitika Sanger (10 , ${ }^{1,2}$ Alessia D'Elia (1) , ${ }^{2,3}$ Stephanie Sanger, ${ }^{4}$ Tea Rosic, ${ }^{2}$ \\ M Constantine Samaan (D) , ${ }^{5}$ Flávio Kapczinski (D) , ${ }^{2}$ Russell J de Souza (D) ,6 \\ Lehana Thabane (D) , ${ }^{6,7,8}$ Zainab Samaan (D) 2,6,9
}

To cite: Sanger N, D'Elia A, Sanger S, et al. Association between vaping and health outcomes in patients with opioid use disorder: a systematic review protocol. BMJ Open 2021;11:e040349. doi:10.1136/ bmjopen-2020-040349

- Prepublication history for this paper is available online. To view these files, please visit the journal online (http://dx.doi org/10.1136/bmjopen-2020040349).

NS and AD contributed equally.

Received 11 May 2020

Revised 11 December 2020

Accepted 17 December 2020

Check for updates

(C) Author(s) (or their employer(s)) 2021. Re-use permitted under CC BY-NC. No commercial re-use. See rights and permissions. Published by BMJ.

For numbered affiliations see end of article.

Correspondence to

Dr Zainab Samaan;

samaanz@mcmaster.ca

\section{ABSTRACT}

Introduction Vaping behaviour has increased in popularity and is particularly important to examine how it effects health outcomes in vulnerable populations, including those with opioid use disorder (OUD). With polysubstance use including cigarette and cannabis use being highly prevalent in the OUD population and cannabis/nicotine increasingly being consumed by vaping, vaping may have an important contribution to health outcomes in these individuals. The primary objective of this review is to systematically assess the literature related to patients with OUD and the effects vaping has shown on their physical and mental health.

Method and analysis A systematic search of databases including MEDLINE, Embase, PsycINFO, Web of Science, Cumulative Index to Nursing and Allied Health Literature, Cochrane Library, Cochrane Clinical Trials Registry, the National Institutes for Health Clinical Trials Registry and the WHO International Clinical Trials Registry Platform from inception to 31 December 2020 will be conducted. Identified citations will be screened by two reviewers to determine eligibility at the title and abstract level, and then at the full text and data extraction phases. Any disagreements in inclusion will be resolved through unblinded discussion by these reviewers, with any remaining disagreements being resolved by a third reviewer. Data collection from eligible studies will be conducted according to the data extraction form tested prior to abstraction. Included studies will be examined for quality and bias and will be meta-analysed where applicable. This protocol is reported in keeping with the Preferred Reporting Items for Systematic Review and Meta-Analysis Protocols guidelines.

Ethics and dissemination The results for this review will be disseminated through publications in peerreviewed journals, posters and presentations at scientific conferences. Additionally, we are collaborating with the Canadian Addiction Treatment Centre clinics to help disseminate the findings for this review. As this is a systematic review, no ethics approval is needed. Review registration number CRD42020178441.

\section{INTRODUCTION}

\section{Rationale}

Vaping behaviour has increased in popularity, raising many questions about short-term and

\section{Strengths and limitations of this study}

- To our knowledge, this will be the first systematic review examining the association between vaping and health outcomes in the opioid use disorder (OUD) population.

- We aim to conduct additional analyses examining mortality, overdoses, hospitalisations and emergency room visits related to vaping.

- This review may be limited in the number of articles identified that are investigating vaping and health outcomes in the OUD population.

long-term health outcomes in at-risk individuals given the scarcity of evidence and methodological investigations conducted within these populations. Vaping is described as inhaling and exhaling a product, which is referred to as a vapour. ${ }^{1}$ Vapours are aerosols derived from the heating of a substance, most typically being comprised of nicotine, flavours, and recently, cannabis. ${ }^{2}{ }^{3}$ One of the most commonly known and used vaping devices is the e-cigarette, which was initially introduced to the public as being a healthier alternative to smoking a combustible cigarette. ${ }^{4}$ However, increasing evidence suggests that e-cigarettes release other toxins such as volatile organic compounds and carbonyls that are dangerous to the individuals vaping have potential for secondhand and thirdhand smoking. ${ }^{56}$

Vaping has gained traction among Canadians as a less harmful alternative to cigarettes. ${ }^{7}$ The number of individuals between 16 and 19 years of age who report having ever vaped increased from $29.3 \%$ to $37.0 \%$ between 2017 and 2018, with the proportion of individuals having vaped 15 days or more within 30 days rising from $2.1 \%$ to $3.6 \%$ in the same time. ${ }^{8}$ While supporters of vaping might suggest increases can be explained by increased uptake of vaping as a substitution 
for cigarettes in a long-range attempt to quit smoking, evidence indicates that the upward trend in vaping is observed most strongly among those who identify as 'never' or 'experimental' smokers. ${ }^{8}$ As vaping substances such as nicotine or cannabis maintains their addictive properties, this behaviour presents a concern for at-risk populations, including those with opioid use disorder (OUD).

Patients with OUD are at greater risk of polysubstance use, as well as physical and mental health comorbidities. ${ }^{9}{ }^{10}$ Both cigarette smoking and cannabis use are highly prevalent in this population, and it remains important to examine the impact of vaping on these patients. Preliminary results from a study investigating pharmacogenetics of OUD in patients receiving medication-assisted treatment (MAT) reported nearly $19.2 \%$ of individuals with OUD report use of vaping products, with the majority being men $(56 \%)$. Nicotine vaping was reported by $74 \%$ of participants; $33 \%$ reported vaping cannabis (tetrahydrocannabinol, cannabidiol, marijuana and shatter). Eleven per cent reported vaping cannabis and nicotine, and $5.5 \%$ reported vaping water or 'flavour'. ${ }^{11}$

The high prevalence of vaping within this population, in addition to a greater susceptibility towards mental and physical health challenges, necessitates further investigation of the impact of vaping on health outcomes. The primary objective of this study is to systematically review literature pertaining to vaping in patients with OUD in order to extract known health outcomes and identify gaps to inform future studies. We hypothesise that vaping will be associated with negative health outcomes in the OUD population.

\section{Objectives}

The objective of this systematic review is to examine and synthesise the literature investigating the association between vaping and various health outcomes in patients with OUD.

Specifically, we aim to:

1. Summarise the literature examining the association between vaping behaviour and health outcomes in patients with OUD (primary outcomes: physical and mental health conditions related to vaping; secondary outcomes: mortality, overdoses, hospitalisations and emergency room visits related to vaping).

2. If possible, pool studies together to conduct a metaanalysis.

3. Conduct subgroup analyses based on age, sex, gender, country, study setting and type of substance vaped and treatment status of OUD (receiving treatment vs not receiving treatment).

\section{METHODS AND ANALYSIS}

This protocol was reported using the Preferred Reporting Items for Systematic Review and Meta-Analysis Protocols (PRISMA-P) guideline. ${ }^{12}$

\section{Eligibility criteria}

The included studies will be randomised control trials (RCT) and observational studies that investigate the association between vaping and health outcomes in the OUD population. We will also include any relevant case studies or case series that will then be qualitatively reported. Studies will be excluded if they are incomplete (eg, ongoing trials and preliminary reports) or are using animal subjects. In the case of multiple RCT papers published under a single trial registration number, we will include the most recent paper that is relevant to the systematic review. We will exclude review papers, editorials, commentaries and conference proceedings/abstracts.

Studies taking place in community-based settings, and in hospital, including inpatient, outpatient and emergency settings, will be assessed. No restrictions will be made on age, sex, gender, language, country, study population or study design.

\section{Outcomes and prioritisation}

The primary objective of this systematic review is to identify the health outcomes associated with vaping in patients with OUD. For example, these outcomes may include physical health conditions such as cardiovascular health, pain conditions, respiratory health (including any acute respiratory syndromes' effects) or mental health conditions such as other substance use disorders, depression and anxiety disorders. The secondary outcomes for this review include mortality, overdoses, emergency room visits and hospitalisations associated with vaping in patients with OUD.

\section{Information sources}

For this review, we will be searching all relevant search engines for RCTs and observational studies including: MEDLINE, Embase, PsycINFO, Web of Science, Cumulative Index to Nursing and Allied Health Literature, Cochrane Library, Cochrane Clinical Trials Registry, the National Institutes for Health Clinical Trials Registry and the WHO International Clinical Trials Registry Platform from inception until 31 December 2020. Additionally, to identify related grey literature, we will search thesis dissertations databases, and reference lists of included studies will be searched for additional articles and resources.

A health sciences librarian (SS) will be consulted when creating the search strategies for the various databases identified. A broad search strategy will be employed to include titles, abstracts and keyword fields. The included terms in the search strategy are related to OUD and vaping. We present the search strategy for one of the included databases (table 1) and will include the complete table of search strategies for all databases in the full review. These databases will be searched from inception to present, without language or demographic constraints and will be limited to human studies. 


\section{Table 1 Search strategy}

\begin{tabular}{|c|c|}
\hline Database & Search strategy \\
\hline \multirow[t]{18}{*}{ MEDLINE } & 1. exp Opioid-Related Disorders/ \\
\hline & 2. exp Heroin Dependence/ \\
\hline & 3. exp Substance-Related Disorders/ \\
\hline & 4. ((opiate* or opioid* or heroin* or \\
\hline & 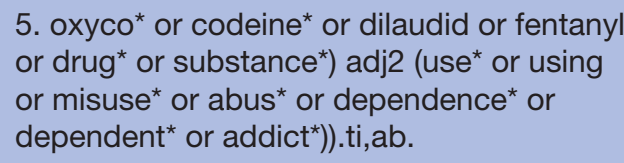 \\
\hline & 6. exp Vaping/ \\
\hline & 7. exp Electronic Nicotine Delivery \\
\hline & 8. Systems/ \\
\hline & 9. electronic nicotine delivery system ${ }^{\star}$ \\
\hline & 10. e-cigarette ${ }^{\star}$ (truncate) \\
\hline & 11. electronic cigarette ${ }^{\star}$ \\
\hline & 12. vape* (get vapes) \\
\hline & 13. vape.mp. \\
\hline & 14. vaping.mp. \\
\hline & 15. e cigarette.mp. \\
\hline & 16. 1 or 2 or 3 or 4 \\
\hline & $\begin{array}{l}17.5 \text { or } 6 \text { or } 7 \text { or } 8 \text { or } 9 \text { or } 10 \text { or } 11 \text { or } 12 \text { or } \\
13\end{array}$ \\
\hline & 18. 14 and 15 limit 16 to humans \\
\hline
\end{tabular}

\section{Data management}

We will use the online platform Covidence to manage references at all stages of the review. ${ }^{13}$ Reviewers will be trained on how to use this tool, and this protocol will be shared with all reviewers. Additionally, we will conduct a calibration stage with 50 articles to ensure team members are in agreement for the review criteria.

\section{Selection process}

All three stages of the review (title and abstract screening, full text screening and data extraction) will be conducted in duplicate. Any disagreements for inclusion of a study will be resolved through unblinded discussion by these reviewers, with any remaining disagreements being resolved by a third, graduate-level reviewer. The recommended flow diagram for systematic reviews in accordance with Preferred Reporting Items for Systematic Reviews and Meta-Analyses guidelines ${ }^{14}$ will be provided, which will list the reasons for inclusion/exclusion. Covidence will be used in all stages of the study selection process including identifying existing duplicates and article organisation. ${ }^{13}$

\section{Data collection process}

The data extraction forms will be created and completed on Covidence and conducted in duplicate. The following items will be collected for each article included for data extraction: author, year, country of origin, population, number of participants (including number of males and females and number assigned to each group of study), control groups used and participant demographic details including mean age and ethnicity. Information on how the authors collected data pertaining to vaping will be noted, including the use of self-report data or validated substance use questionnaires and type of health outcome reported. For all outcomes, we will also note how they are defined, measured and the frequency of measurement. If information from an included study is missing, we will contact the authors to obtain that information and keep a $\log$ of any correspondence.

\section{Risk of bias in individual studies}

All studies included in the data extraction phase will be assessed for risk of bias using appropriate tools corresponding to study design. Such assessments will be completed in duplicate. For RCTs, we will use the Cochrane Risk of Bias Tool, ${ }^{15}$ and for observational studies, we will use the Newcastle-Ottawa Scale. ${ }^{16}$ If possible, on papers that have scored a low risk of bias, we will conduct a sensitivity analysis.

\section{Data synthesis}

The identified RCTs and observational studies will be qualitatively reported. If possible, we will conduct metaanalyses for our primary and secondary outcomes, pooling studies with similar study designs and outcome measurements and presenting results using forest plots. We will use a random-effects model that will take betweenstudy and within-study variance into account as opposed to a fixed-effect model to account for expected heterogeneity. We will assess heterogeneity using the $I^{2}$ statistic and 95\% CI. We will use the Cochrane recommendation of a $I^{2}$ statistic $>40 \%$ indicating high heterogeneity. ${ }^{17}$ Anticipated sources of heterogeneity include age, sex, treatment status and country, for which we will conduct subgroup analyses. We will also conduct subgroup analyses based on the type of substance that is vaped (ie, cannabis, nicotine and water flavour). All quantitative analyses will be conducted on RevMan V.5.3. ${ }^{18}$ Any identified case reports or case series will be qualitatively summarised. If a metaanalysis is not possible (ie, small number of studies and high heterogeneity), we will present a narrative synthesis of the included papers.

\section{Metabias(es)}

To address any publication bias, we will conduct an Eggers test if appropriate.

\section{Data statement}

Any additional data can be made available on request when the review is complete.

\section{Confidence in cumulative evidence}

We will use the grading of recommendations, assessment, development and evaluation framework to assess the quality of the evidence. ${ }^{19}$ This framework will assess the risk of bias, publication bias, consistency, directness and accuracy of the literature. 


\section{Patient and public involvement}

There will be no patient or public involvement for this systematic review.

\section{ETHICS AND DISSEMINATION}

The completed systematic review will have a knowledge dissemination component. We will have clinicians, researchers and people that vape as users of this information. We will collaborate with the Canadian Addiction Treatment Centres, an organisation that delivers MAT for the OUD population, to disseminate our findings. We plan to make our findings available to healthcare professionals in various settings through summary reports and resources that will be convenient to access. We will also publish this systematic review in a peer-reviewed journal and present our findings in relevant conferences to the scientific community. No ethics approval is needed as this is a systematic review looking at published literature.

\section{Author affiliations}

${ }^{1}$ Medical Science Program, McMaster University Faculty of Health Sciences, Hamilton, Ontario, Canada

${ }^{2}$ Department of Psychiatry and Behavioural Neurosciences, McMaster University, Hamilton, Ontario, Canada

${ }^{3}$ Neuroscience Graduate Program, McMaster University, Hamilton, Ontrio, Canada ${ }^{4}$ Health Sciences Library, McMaster University Faculty of Health Sciences, Hamilton, Ontario, Canada

${ }^{5}$ Division of Pediatric Endocrinology, McMaster Children's Hospital, Hamilton, Ontario, Canada

${ }^{6}$ Department of Health Research Methods, Evidence and Impact, McMaster University, Hamilton, Ontario, Canada

${ }^{7}$ Population Health Research Institute, Hamilton, Ontario, Canada

${ }^{8}$ Father Sean 0'Sullivan Research Centre, St. Joseph's Healthcare, Hamilton, Ontario, Canada

${ }^{9}$ Department of Psychiatry and Behavioral Neurosciences, Faculty of Health Sciences, McMaster University, Hamilton, Ontario, Canada

Correction notice This article has been corrected since it first published. The provenance and peer review statement has been included.

Contributors NS and AD: contributed to the conception and design of the study, development of data extraction forms, search strategy, manuscript writing and final review of the manuscript. SS: contributed to the development of the search strategy and final review of the manuscript. FK, MCS, RJdS and LT: contributed to the critical revision and final review of the manuscript. ZS: contributed to the conception and design of the study, critical revision and final approval of the manuscript. All authors read and approved the final manuscript.

Funding ZS is supported by grants from CIHR Awards \#156306 and \#155404.

Competing interests None declared.

Patient and public involvement Patients and/or the public were not involved in the design, or conduct, or reporting, or dissemination plans of this research.

Patient consent for publication Not required.

Provenance and peer review Not commissioned; externally peer reviewed.

Open access This is an open access article distributed in accordance with the Creative Commons Attribution Non Commercial (CC BY-NC 4.0) license, which permits others to distribute, remix, adapt, build upon this work non-commercially, and license their derivative works on different terms, provided the original work is properly cited, appropriate credit is given, any changes made indicated, and the use is non-commercial. See: http://creativecommons.org/licenses/by-nc/4.0/.

\section{ORCID iDs}

Nitika Sanger http://orcid.org/0000-0002-5883-1873

Alessia D'Elia http://orcid.org/0000-0002-6512-4704

M Constantine Samaan http://orcid.org/0000-0002-6403-4715

Flávio Kapczinski http://orcid.org/0000-0001-8738-856X

Russell J de Souza http://orcid.org/0000-0001-8945-513X

Lehana Thabane http://orcid.org/0000-0003-0355-9734

Zainab Samaan http://orcid.org/0000-0002-5974-9361

\section{REFERENCES}

1 Qasim H, Karim ZA, Rivera JO, et al. Impact of electronic cigarettes on the cardiovascular system. J Am Heart Assoc 2017;6:e006353.

2 Budney AJ, Sargent JD, Lee DC. Vaping cannabis (marijuana): parallel concerns to e-cigs? Addiction 2015;110:1699-704.

3 Young-Wolff KC, Klebaner D, Folck B, et al. Do you vape? Leveraging electronic health records to assess clinician documentation of electronic nicotine delivery system use among adolescents and adults. Prev Med 2017;105:32-6.

4 McRobbie H, Bullen C, Hartmann-Boyce J. Electronic cigarettes for smoking cessation and reduction. Cochrane Database Syst Rev 2014;12.

5 Cheng T. Chemical evaluation of electronic cigarettes. Tob Control 2014;23 Suppl 2:ii11-17.

6 Farsalinos KE, Gillman IG, Melvin MS, et al. Nicotine levels and presence of selected tobacco-derived toxins in tobacco flavoured electronic cigarette refill liquids. Int J Environ Res Public Health 2015;12:3439-52.

7 Public health consequences of e-cigarettes 2018.

8 Hammond D, Reid JL, Rynard VL, et al. Prevalence of vaping and smoking among adolescents in Canada, England, and the United States: repeat national cross sectional surveys. BMJ 2019;365:I2219.

9 Fischer Bet al. Illicit opioid use in Canada: comparing social, health, and drug use characteristics of untreated users in five cities (OPICAN study). J Urban Heal 2005;82:250-66.

10 Sanger N, Bhatt M, Shams I. Association between sociodemographic and health functioning variables among patients with opioid use disorder introduced by prescription: a prospective cohort study, 2018: 623-32.

11 Samaan Z, MacKillop J, Pare G. Pharmacogenetics of opioid substitution treatment, 2018.

12 Moher D, Shamseer L, Clarke M, et al. Preferred reporting items for systematic review and meta-analysis protocols (PRISMA-P) 2015 statement. Syst Rev 2015;4:1

13 Innovation VH. Covidence systematic review software, 2017. Available: www.covidence.org

14 Moher D, Liberati A, Tetzlaff J, et al. Preferred reporting items for systematic reviews and meta-analyses: the PRISMA statement. PLOS Med 2009;6:e1000097.

15 Higgins JPT, Altman DG, Gøtzsche PC, et al. The Cochrane collaboration's tool for assessing risk of bias in randomised trials. BMJ 2011;343:d5928.

16 Wells GA, Shea B, O'connell D. The Newcastle-Ottawa Scale (NOS) for assessing the quality if nonrandomized studies in meta-analyses, 2009. Available: http//www ohri ca/programs/clinical_epidemiology/ oxford htm

17 Higgins JPT, Green S. Cochrane Handbook for systematic reviews of interventions. Vol 4. John Wiley \& Sons, 2011.

18 Collaboration C. Review manager (version 5.3)[computer software]. Copenhagen. Denmark Nord Cochrane Centre, Cochrane Collab, 2014.

19 Guyatt GH, Oxman AD, Schünemann HJ, et al. Grade guidelines: a new series of articles in the Journal of clinical epidemiology. $J$ Clin Epidemiol 2011;64:380-2. 